ANNALES

POLONICI MATHEMATICI

LXXVIII.3 (2002)

\title{
Periodic solutions of $n$th order delay Rayleigh equations
}

\author{
by Gen-Qiang Wang (Guangzhou) and Sui Sun Cheng (Hsinchu)
}

Abstract. A priori bounds are established for periodic solutions of an $n$th order Rayleigh equation with delay. From these bounds, existence theorems for periodic solutions are established by means of Mawhin's continuation theorem.

In [4], a priori bounds for periodic solutions of the equation

$$
x^{\prime \prime}(t)+\lambda f\left(x^{\prime}(t)\right)+\lambda g(x(t-\tau(t)))=\lambda p(t), \quad \lambda \in(0,1),
$$

are established under relatively simple conditions on $f, g$ and $p$. Then by means of continuation theorems [1], periodic solutions for the Rayleigh differential equation

$$
x^{\prime \prime}(t)+f\left(x^{\prime}(t)\right)+g(x(t-\tau(t)))=0
$$

are obtained.

In this note, we will be concerned with similar equations of the form

$$
\begin{aligned}
x^{(n)}(t)+\lambda f\left(t, x^{\prime}\left(t-\tau_{1}(t)\right), \ldots, x^{(n-1)}(\right. & \left.\left.t-\tau_{n-1}(t)\right)\right) \\
& +\lambda g\left(t, x\left(t-\tau_{0}(t)\right)\right)=\lambda p(t),
\end{aligned}
$$

and

$$
\begin{aligned}
x^{(n)}(t)+f\left(t, x^{\prime}\left(t-\tau_{1}(t)\right), \ldots, x^{(n-1)}(t-\right. & \left.\left.\tau_{n-1}(t)\right)\right) \\
& +g\left(t, x\left(t-\tau_{0}(t)\right)\right)=p(t)
\end{aligned}
$$

where $\lambda \in(0,1), n \geq 2, \tau_{0}, \ldots, \tau_{n-1}$ and $p$ are $T$-periodic continuous functions defined on $\mathbb{R}$ with

$$
\int_{0}^{T} p(t) d t=0,
$$

$f$ is continuous on $\mathbb{R}^{n}, f(t, 0, \ldots, 0)=0$ for $t \in \mathbb{R}$ and $f\left(t+T, x_{1}, \ldots, x_{n-1}\right)$ $=f\left(t, x_{1}, \ldots, x_{n-1}\right)$ for $\left(t, x_{1}, \ldots, x_{n-1}\right) \in \mathbb{R}^{n}$, and $g$ is continuous on $\mathbb{R}^{2}$

2000 Mathematics Subject Classification: 34K10, 34K13.

Key words and phrases: Rayleigh equation, delay, periodic solution. 
such that $g(t+T, x)=g(t, x)$ for $(t, x) \in \mathbb{R}^{2}$. To avoid trivial cases, we also assume that the period $T$ is positive.

We will establish a priori bounds for periodic solutions of equation (2) under several conditions imposed on $f$ and $g$. Once these bounds are obtained, existence of periodic solutions for equation (3) can be demonstrated.

We remark that there are a number of studies which are concerned with the existence of periodic solutions of Rayleigh differential equations (see e.g. $[2,3,5])$. But our conditions are novel and relatively simple as compared to many others. For example, in [3], smoothness in addition to boundedness assumptions are needed for the functions in (1) in order to guarantee a periodic solution.

TheOREm 1. Suppose there are constants $H \geq 0, D>0$ and $M>0$ such that

(i) $\left|f\left(t, x_{1}, \ldots, x_{n-1}\right)\right| \leq H$ for $\left(t, x_{1}, \ldots, x_{n-1}\right) \in \mathbb{R}^{n}$,

(ii) $x g(t, x)>0$ and $|g(t, x)|>H$ for $t \in \mathbb{R}$ and $|x| \geq D$, and

(iii) $|g(t, x)| \leq M$ for $t \in \mathbb{R}$ and $x \leq-D$.

Then there exist $D_{0}, \ldots, D_{n-1}>0$ such that for any T-periodic solution $x=x(t)$ of $(2)$,

$$
\left|x^{(j)}(t)\right| \leq D_{j}, \quad 0 \leq j \leq n-1,0 \leq t \leq T .
$$

Proof. Let $x=x(t)$ be a $T$-periodic solution of (2). In view of (2), and the periodicity of $x(t)$,

$$
\int_{0}^{T}\left\{f\left(t, x^{\prime}\left(t-\tau_{1}(t)\right), \ldots, x^{(n-1)}\left(t-\tau_{n-1}(t)\right)\right)+g\left(t, x\left(t-\tau_{0}(t)\right)\right)\right\} d t=0 .
$$

Note also that

$$
\int_{0}^{T}\left|f\left(t, x^{\prime}\left(t-\tau_{1}(t)\right), \ldots, x^{(n-1)}\left(t-\tau_{n-1}(t)\right)\right)\right| d t \leq T H .
$$

Thus,

$$
\begin{aligned}
& \int_{0}^{T}\left\{g\left(t, x\left(t-\tau_{0}(t)\right)\right)-H\right\} d t \\
\leq & \int_{0}^{T}\left\{g\left(t, x\left(t-\tau_{0}(t)\right)\right)-\left|f\left(t, x^{\prime}\left(t-\tau_{1}(t)\right), \ldots, x^{(n-1)}\left(t-\tau_{n-1}(t)\right)\right)\right|\right\} d t \\
\leq & \int_{0}^{T}\left\{g\left(t, x\left(t-\tau_{0}(t)\right)\right)+f\left(t, x^{\prime}\left(t-\tau_{1}(t)\right), \ldots, x^{(n-1)}\left(t-\tau_{n-1}(t)\right)\right)\right\} d t=0 .
\end{aligned}
$$


Let

$$
\begin{array}{ll}
G_{+}(t)=\max \left\{g\left(t, x\left(t-\tau_{0}(t)\right)\right)-H, 0\right\}, & t \in \mathbb{R}, \\
G_{-}(t)=\max \left\{H-g\left(t, x\left(t-\tau_{0}(t)\right)\right), 0\right\}, & t \in \mathbb{R} .
\end{array}
$$

Then $G_{+}$and $G_{-}$are nonnegative and continuous on $\mathbb{R}$,

$$
g\left(t, x\left(t-\tau_{0}(t)\right)\right)-H=G_{+}(t)-G_{-}(t), \quad t \in \mathbb{R},
$$

and in view of (ii) and (iii),

$$
G_{-}(t)=\left|G_{-}(t)\right| \leq H+M, \quad t \in \mathbb{R} .
$$

In view of (6) and (7), we have

$$
\int_{0}^{T} G_{+}(t) d t \leq \int_{0}^{T} G_{-}(t) d t \leq M_{1}
$$

where $M_{1}=(H+M) T$. In view of $(7)$, we have

$$
\int_{0}^{T}\left|g\left(t, x\left(t-\tau_{0}(t)\right)\right)-H\right| d t \leq 2 M_{1}
$$

which implies

$$
\int_{0}^{T}\left|g\left(t, x\left(t-\tau_{0}(t)\right)\right)\right| d t \leq 2 M_{1}+T H .
$$

By integrating (2), in view of (5) and (8), we see that

$$
\begin{aligned}
\int_{0}^{T}\left|x^{(n)}(t)\right| d t \leq & \int_{0}^{T}\left|f\left(t, x^{\prime}\left(t-\tau_{1}(t)\right), \ldots, x^{(n-1)}\left(t-\tau_{n-1}(t)\right)\right)\right| d t \\
& +\int_{0}^{T}\left|g\left(t, x\left(t-\tau_{0}(t)\right)\right)\right| d t+\int_{0}^{T}|p(t)| d t \\
\leq & T H+2 M_{1}+T H+T \max _{0 \leq t \leq T}|p(t)| .
\end{aligned}
$$

Since $x^{(n-2)}(0)=x^{(n-2)}(T)$, there exists $t_{1} \in[0, T]$ such that $x^{(n-1)}\left(t_{1}\right)$ $=0$. Thus

$$
\left|x^{(n-1)}(t)\right|=\left|\int_{t_{1}}^{t} x^{(n)}(s) d s\right| \leq \int_{0}^{T}\left|x^{(n)}(s)\right| d s \leq D_{n-1}, \quad t \in[0, T],
$$

where $D_{n-1}=T H+2 M_{1}+T H+T \max _{0 \leq t \leq T}|p(t)|>0$. Next we will show that when $n>2$, we have $\left|x^{(j)}(t)\right| \leq \bar{D}_{j}$ for $1 \leq j \leq n-2$ and $0 \leq t \leq T$. Indeed, since $x^{(n-3)}(0)=x^{(n-3)}(T)$, there exists $t_{2} \in[0, T]$ such 
that $x^{(n-2)}\left(t_{2}\right)=0$. As a consequence,

$$
\left|x^{(n-2)}(t)\right|=\left|\int_{t_{2}}^{t} x^{(n-1)}(s) d s\right| \leq \int_{0}^{T}\left|x^{(n-1)}(s)\right| d s \leq T D_{n-1} \equiv D_{n-2} .
$$

The rest of the proof follows by induction. To complete our proof, we will show that $|x(t)| \leq D_{0}, t \in[0, T]$, for some $D_{0}>0$. Indeed, in view of (4),

$$
f\left(t_{3}, x^{\prime}\left(t_{3}-\tau_{1}\left(t_{3}\right)\right), \ldots, x^{(n-1)}\left(t_{3}-\tau_{n-1}\left(t_{3}\right)\right)\right)+g\left(t_{3}, x\left(t_{3}-\tau_{0}\left(t_{3}\right)\right)\right)=0
$$

for some $t_{3} \in[0, T]$. Hence by $(\mathrm{i})$,

$\left|g\left(t_{3}, x\left(t_{3}-\tau_{0}\left(t_{3}\right)\right)\right)\right|=\left|f\left(t_{3}, x^{\prime}\left(t_{3}-\tau_{1}\left(t_{3}\right)\right), \ldots, x^{(n-1)}\left(t_{3}-\tau_{n-1}\left(t_{3}\right)\right)\right)\right| \leq H$.

But then by (ii), $\left|x\left(t_{3}-\tau_{0}\left(t_{3}\right)\right)\right|<D$. Since $x(t)$ is $T$-periodic, there exists $t_{4} \in[0, T]$ such that $\left|x\left(t_{4}\right)\right|<D$. Finally,

$$
|x(t)|=\left|x\left(t_{4}\right)+\int_{t_{4}}^{t} x^{\prime}(s) d s\right| \leq D+\int_{0}^{T}\left|x^{\prime}(s)\right| d s \leq D+T D_{1}
$$

for $t \in[0, T]$. The proof is complete.

Having the a priori bounds just obtained, we may follow the standard procedures as explained in various places of [1] and the continuation theorem on page 40 of [1] to show the existence of a periodic solution of (3). For completeness, a brief sketch is included.

Let $X$ be the Banach space of all functions $x=x(t) \in C^{(n-1)}(\mathbb{R})$ such that $x(t+T)=x(t)$ for all $t$, endowed with the norm

$$
\|x\|=\sum_{j=0}^{n-1} \max _{0 \leq t \leq T}\left|x^{(j)}(t)\right| \text {. }
$$

Also let $Y$ be the Banach space of all continuous functions of the form $y=y(t)$ defined on $\mathbb{R}$ such that $y(t+T)=y(t)$ for all $t$, and endowed with the norm $\|y\|_{0}=\max _{0 \leq t \leq T}|y(t)|$. Now let $L: X \cap C^{(n)}(\mathbb{R}) \rightarrow Y$ be the operator defined by $(L \bar{x})(t)=x^{(n)}(t)$ for $t \in \mathbb{R}$, and let $N: X \rightarrow Y$ be defined by

$(N x)(t)=-f\left(t, x^{\prime}\left(t-\tau_{1}(t)\right), \ldots, x^{(n-1)}\left(t-\tau_{n-1}(t)\right)\right)-g\left(t, x\left(t-\tau_{0}(t)\right)\right)+p(t)$

for $t \in \mathbb{R}$. Let $\operatorname{Im} L$ and $\operatorname{Ker} L$ be respectively the image and kernel of the operator $L$. Clearly, $\operatorname{Ker} L=\mathbb{R}$. Furthermore, if we define the projections $P: X \rightarrow \operatorname{Ker} L$ and $Q: Y \rightarrow Y / \operatorname{Im} L$ by

$$
(P x)(t)=\frac{1}{T} \int_{0}^{T} x(t) d t, \quad(Q y)(t)=\frac{1}{T} \int_{0}^{T} y(t) d t, \quad t \in \mathbb{R},
$$

then $\operatorname{Ker} L=\operatorname{Im} P$ and $\operatorname{Ker} Q=\operatorname{Im} L$. Furthermore, $L$ is a Fredholm operator of index zero. The operator $N$ is continuous and maps bounded subsets 
of $X$ into bounded subsets of $Y$, thus for any bounded open subset $\Omega$ of $X, N(\bar{\Omega})$ is bounded. This shows that $(I-Q) N(\bar{\Omega})$ is bounded. Since the inverse $K$ of $L_{\mid \operatorname{dom} L \cap \operatorname{ker} P}$ is compact, $K(I-Q) N(\bar{\Omega})$ is relatively compact, and so $L$-compact on the closure of $\Omega$ (see e.g. [1, pp. 166-187]).

Let $D, D_{0}, \ldots, D_{n-1}$ be as in Theorem 1 , and let $\Omega$ be the subset of $X$ consisting of the functions of the form $x=x(t)$ such that $\|x\|<\bar{D}$, where $\bar{D}$ is a fixed number which satisfies $\bar{D}>\max \left\{D_{0}, D_{1}, \ldots, D_{n-1}\right\}+D$. For any $\lambda \in(0,1)$ and any $x=x(t)$ in the domain of $L$ which also belongs to $\partial \Omega$, we must have $L x \neq \lambda N x$. For otherwise in view of $\|x\|<\bar{D}, x$ belongs to the interior of $\Omega$, contrary to the assumption that $x \in \partial \Omega$. Next, note that a function $x=x(t) \in \operatorname{Ker} L \cap \partial \Omega$ must be the constant function $x(t) \equiv \bar{D}$ or $x(t) \equiv-\bar{D}$. Hence

$$
\begin{aligned}
(Q N)(x)= & \frac{1}{T} \int_{0}^{T}\left[-f\left(t, x^{\prime}\left(t-\tau_{1}(t)\right), \ldots, x^{(n-1)}\left(t-\tau_{n-1}(t)\right)\right)\right] d t \\
& +\frac{1}{T} \int_{0}^{T}\left[-g\left(t, x\left(t-\tau_{0}(t)\right)+p(t)\right] d t\right. \\
= & \frac{1}{T} \int_{0}^{T}\left[-f(t, 0, \ldots, 0)-g\left(t, x\left(t-\tau_{0}(t)\right)\right)\right] d t \\
= & -\frac{1}{T} \int_{0}^{T} g\left(t, x\left(t-\tau_{0}(t)\right)\right) d t=-\frac{1}{T} \int_{0}^{T} g(t, x) d t \neq 0 .
\end{aligned}
$$

Finally, consider the mapping

$$
H(x, s)=s x+(1-s) \frac{1}{T} \int_{0}^{T} g(t, x) d t, \quad 0 \leq s \leq 1 .
$$

Since for every $s \in[0,1]$ and $x \in \operatorname{Ker} L \cap \partial \Omega$, we have

$$
x H(x, s)=s x^{2}+(1-s) x \frac{1}{T} \int_{0}^{T} g(t, x) d t>0,
$$

$H(x, s)$ is an admissible homotopy. This shows that

$$
\begin{aligned}
\operatorname{deg}\{Q N x, \Omega \cap \operatorname{Ker} L, 0\} & =\operatorname{deg}\left\{-\frac{1}{T} \int_{0}^{T} g(t, x) d t, \Omega \cap \operatorname{Ker} L, 0\right\} \\
& =\operatorname{deg}\{-x, \Omega \cap \operatorname{Ker} L, 0\} \\
& =\operatorname{deg}\{-x, \Omega \cap \mathbb{R}, 0\} \neq 0 .
\end{aligned}
$$

We have thus verified all the assumptions of the continuation theorem $[1$, p. 40]. Under the assumptions of Theorem 1, equation (3) thus has a $T$ periodic solution. 
TheOREm 2. Suppose the assumptions of Theorem 1 hold. Then equation (3) has a T-periodic solution.

As an example, consider the equation

$$
\begin{aligned}
x^{\prime \prime \prime}(t)+\exp \left\{-\sin ^{2} t-\left(x^{\prime}(t-\cos t)\right.\right. & )^{2}-\left(x^{\prime \prime}(t-\sin t)\right)^{2}\right\} \\
& \left.+\left(1+\cos ^{2} t\right) \arctan (x(t-\sin t))\right) \\
= & \sin t+\exp \left(-\sin ^{2} t\right) .
\end{aligned}
$$

Take

$$
\begin{aligned}
f\left(t, x_{1}, x_{2}\right) & =\exp \left(-\sin ^{2} t-x_{1}^{2}-x_{2}^{2}\right)-\exp \left(-\sin ^{2} t\right), \\
g(t, x) & =\left(1+\cos ^{2} t\right) \arctan x,
\end{aligned}
$$

$\tau_{0}(t)=\sin t, \tau_{1}(t)=\cos t, \tau_{2}(t)=\sin t$, and $p(t)=\sin t$ and $T=2 \pi$. It is then easy to verify that all the assumptions of Theorem 1 are satisfied with $H=1, D>\pi / 4$ and $M=\pi$. Hence this equation has a $2 \pi$-periodic solution.

We remark that by symmetric arguments, we can establish the following existence theorem.

TheOREm 3. Suppose there are constants $H \geq 0, D>0$ and $M>0$ such that (i) $\left|f\left(t, x_{1}, \ldots, x_{n-1}\right)\right| \leq H$ for $\left(t, x_{1}, \ldots, x_{n-1}\right) \in \mathbb{R}^{n}$, (ii) $x g(t, x)>0$ and $|g(t, x)|>H$ for $t \in \mathbb{R}$ and $|x| \geq D$, and (iii) $|g(t, x)| \leq M$ for $t \in \mathbb{R}$ and $x \geq D$. Then (3) has a T-periodic solution.

\section{References}

[1] R. E. Gaines and J. L. Mawhin, Coincidence Degree and Nonlinear Differential Equations, Lecture Notes in Math. 568, Springer, 1977.

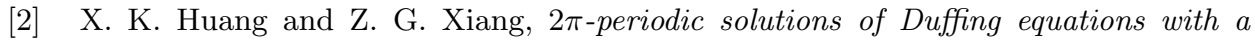
deviating argument, Chinese Sci. Bull. 39 (1994), 201-203.

[3] S. Invernizzi and F. Zanolin, Periodic solutions of a differential delay equation of Rayleigh type, Rend. Sem. Mat. Univ. Padova 61 (1979), 115-124.

[4] G. Q. Wang and S. S. Cheng, A priori bounds for periodic solutions of a delay Rayleigh equation, Appl. Math. Lett. 12 (1999), 41-44.

[5] G. Q. Wang and J. R. Yan, Existence of periodic solutions for nth order nonlinear delay differential equation, Far East J. Appl. Math. 3 (1999), 129-134.

Department of Computer Science

Guangdong Polytechnical Normal University

Guangzhou, Gaungdong 510665, P.R. China
Department of Mathematics

Tsing Hua Univeristy Hsinchu, Taiwan 30043, R.O.C. E-mail: sscheng@math.nthu.edu.tw 